\title{
Estenose de carótida: por que defendo o tratamento endovascular
}

\author{
Carotid stenosis. why I support the endovascular treatment
}

\author{
Walter Karakhanian*
}

\begin{abstract}
A avaliação dos resultados, a curto e a longo prazo, na mudança de uma conduta médica, ésempre difícil e delicada. I sso é particularmente verdadeiro no tratamento das estenoses de carótidas, onde existe a endarterectomia, considerada padrão-ouro nesse tipo de doença. A endarterectomia decarótida sofreu o julgamento do tempo por cinco décadas ehá muito faz parte do arsenal terapêutico do cirurgião vascular. Porém, na era da medicina minimamente invasiva, o padrão-ouro começa a ser desafiado.

A defesa aqui apresentada será fundamentada em doiscritérios: o primeiro, maisobjetivo, estábaseado no nível científico de evidências segundo os critérios de
\end{abstract}

Sackett ${ }^{1}$ (a relação entre 0 nível de evidências e as recomendações clínicas está resumido na tabela abaixo); o segundo, mais subjetivo, é de al guém que vivenciou e vivencia as duas técnicas (T abela 1).

As conclusões dos estudos clássicos com evidências I ell no tratamento endovascular da doença carotídea, consideran do a eficácia ea segurança desseprocedimento, são descritas a seguir.

CAVAT AS ${ }^{2}$. Estudo multicêntrico randomizado com 504 pacientes, sem dispositivo de proteção estent em apenas $26 \%$ dos casos: "A endarterectomia de carótida e o tratamento endovascular são equival entes quanto à eficácia e segurança no queserefere às compli-

T abela 1 - Primeiro critério: medicina baseada em evidências

\begin{tabular}{|c|c|c|}
\hline \multicolumn{2}{|c|}{ N ível de evidências } & \multirow{2}{*}{$\begin{array}{c}\text { Grau de recomendação clínica } \\
\text { A }\end{array}$} \\
\hline I & $\begin{array}{l}\text { - Estudo randomizado com grande número de doentes } \\
\text { Resultados claros }\end{array}$ & \\
\hline II & $\begin{array}{l}\text { - Estudo randomizado com pequeno número de doentes } \\
\text { Resultados incertos }\end{array}$ & $B$ \\
\hline III & - Estudo não-randomizado & C \\
\hline IV & - Estudo não-randomizado & C \\
\hline V & - Estudo não-controlado & $\mathrm{C}$ \\
\hline
\end{tabular}

\footnotetext{
* Professor assistente, Faculdade de Ciências Médicas, Santa Casa de São Paulo, São Paulo, SP 
cações maiores - acidente vascular cerebral (AVC), infarto do miocárdio emorte- ena prevenção denovos episódios de isquemia ipsolateral. Q uando avaliadas as lesões de nervos e hematomas das vias de acesso, houve uma diferença significativa em favor do grupo endovascular $(p<0,0001)^{\prime \prime}$.

LEXIN GT O N ${ }^{3}$. Estudo randomizado com 104 pacientes sintomáticose com estenose $>70 \%$ - critério $\mathrm{N}$ orth American Symptomatic Carotid Endaterectomy Trial (N ASCET) -, tratados com endarterectomia e stent sem dispositivo deproteção: "O tratamento endovascular não aumenta os riscos de AVC ou morte quando comparado ao tratamento cirúrgico".

SAPPH IRE 4 . Estudo randomizado, envolvendo pacientes dealto risco com estenose de carótida. Foram incluídos pacientes sintomáticos com estenose $>$ que $50 \%$ e assintomáticos com estenose $>$ que $80 \%$. Todas as angioplastias foram feitas com dispositivo de proteção cerebral (Angioguard ${ }^{\circledR}$ - Cordis). A avaliação foi feita em 30 diase 1 ano, tendo como objetivo avaliar 0 AVC do mesmo lado, o infarto do miocárdio eo óbito. C oncluiu-se que "a angioplastia com stent não é inferior à endarterectomia".

LEICEST ER ${ }^{5}$. Estudo suspenso após a inclusão de 17 pacientes pelo elevado índice de complicações no braço endovascular.

Com relação aos relatos de autores (níveis de evidênciallI elV ), observaram-se as seguintes conclusões: índice de complicações do tratamento endovascular bastante aceitável (Roubin et al. ${ }^{6}$ ); importância da curva de aprendizado (A hmadi et al. ${ }^{7}$ ); ealternativa ao tratamento cirúrgico convencional, principalmenteem pacientes deal to risco ecom perspectiva demelhoria em função do avanço tecnológico dos materiais (W holey et al. $\left.{ }^{8}\right)$.

Portanto, há evidências apontando para uma provável mudança na abordagem terapêutica da doença da bifurcação carotídea em direção ao tratamento endovascular.

Levando-se em conta o segundo critério, isto é, o subjetivo, gostaria de tecer algumas considerações:

- Durante 24 anos, participei, e ainda participo, da formação de residentes na Faculdade de Ciências M édicas da Santa C asa de São Paulo e observei a evolução demuitos delesno quesito "endarterectomia decarótida". A partir do ano de2000, o Serviço de Radiologia $V$ ascular foi efetivamente implanta- do no hospital. D esde então, dediquei-me ao ensino do tratamento endovascular. $\mathrm{H}$ oje, concluo, como observação pessoal, quea curva de aprendizado dessa forma de tratamento é muito mais rápida que a da endarterectomia. Em outras palavras, 0 médico residente atinge 0 nível de excelência em muito menos tempo do quena cirurgia convencional. Todavia, devo salientar que faz parte desse treinamento a prática intensiva do diagnóstico em angiografia cerebral, com o conhecimento detécnicas básicas de cateterismo, de materiais e das anatomias extra e intracraniana.

- A outra consideração é que, nesses anos, vivenciando os resultados da cirurgia aberta eos resultados do tratamento endovascular, acredito que as complicações maiores, como AVC e morte, são muito semel hantes entre as duas técnicas. Por outro lado, as complicações "menores" são muito menos freqüentes nas angioplastias.

- N os pacientes submetidos às duas formas de tratamento, ambas sem intercorrências, não há dúvida de que a preferência é pelo tratamento endovascular.

Portanto, diantedessesfatoseargumentos, defendo o tratamento endovascular na doença aterosclerótica da bifurcação carotídea extracraniana, acreditando que veio para ficar esubstituir a endarterectomia namaioria dos pacientes. N unca é demais ressaltar que, para isso, o profissional deve estar apto a fazêla, passando pelo treinamento adequado.

0 cirurgião vascular deveoptar pelo tratamento no qual se sente mais confortável e com menor probabilidade de prejudicar o seu doente.

\section{R eferências}

1. Sackett DL. Rules of evidence and clinical recommendations on the use of antithrombotic agents. Chest. 1989;95 2 Suppl.:2S-4S.

2. Endovascular versussurgical treatment in patients with carotid stenosis in the Carotid and Vertebral Artery Transluminal Angioplasty Study (CAVATAS): a randomised trial. Lancet. 2001:357:1729-37.

3. BrooksW H, M cClureR R, JonesM R, Coleman T C, Breathitt L. Carotid angioplasty and stenting versus carotid endarterectomy: randomized trial in a community hospital. J Am Coll Cardiol. 2001;38:1589-95.

4. Yadav JS, W holey M H, Kuntz RE, et al. Protected carotidartery stenting versus endarterectomy in high-risk patients. $\mathrm{N}$ Engl J M ed. 2004;351:1493-501. 
5. N aylor AR, Bolia A, Abbott RJ, et al. Randomized study of carotid angioplasty and stenting versuscarotid endarterectomy: a stopped trial. J Vasc Surg. 1998;28:326-34.

6. Roubin GS, N ew G, Iyer SS, et al. I mmediateand lateclinical outcomes of carotid artery stenting in patients with symptomatic carotid artery stenosis: a 5-year prospective analysis. Circulation. 2001;103:532-7.

7. A hmadi R, W illfort $A$, Lang $W$, et al. C arotid artery stenting: effect of learning curve and intermediate-term morphological outcome. J Endovasc Ther. 2001;8:539-46.

8. Wholey M H , Al- M ubarek N, W holey M H . U pdatereview of the global carotid artery stent registry. Catheter $\mathrm{C}$ ardiovasc Interv. 2003:60:259-66.

\section{Aviso aos ex-residentes}

Se você foi residente até 2005 e ainda não está inscrito em sua Regional, procure regularizar sua situação para passar a receber o J Vasc Bras imediatamente, sem qualquer ônus.

Ajude a manter o cadastro da SBACV atualizado. 